\title{
An autophagy inhibitor enhances the inhibition of cell proliferation induced by a proteasome inhibitor in MCF-7 cells
}

\author{
FENG YAO*, GUANNAN WANG* , WEN WEI, YI TU, HEXIANG TONG and SHENGRONG SUN \\ Department of Breast and Thyroid Surgery, Renmin Hospital of Wuhan University, Wuhan, Hubei, P.R. China
}

Received June 28, 2011; Accepted September 14, 2011

DOI: $10.3892 / \mathrm{mmr} .2011 .590$

\begin{abstract}
The ubiquitin-proteasome system and the autophagy-lysosome pathway are the two main routes for eukaryotic intracellular protein clearance. Inhibition of proteasome activity leads to cell death. Due to the dual roles of autophagy in tumor cell survival and death, the effect of suppression of autophagy on breast cancer cells remains to be elucidated. We investigated whether inhibition of the proteasome is capable of inducing autophagy, and we assessed the effect of combined inhibition of the proteasome and autophagy on human breast cancer MCF-7 cells. The proteasome inhibitor bortezomib was used to induce autophagy in MCF-7 cells, and the effect of autophagy on the proliferation of MCF-7 cells was investigated using the autophagy inhibitor 3-MA. Cell viability was measured by MTT assay. The expression of autophagy-related proteins was determined by Western blot analysis and the GFP-LC3 redistribution was detected using a fluorescence microscope after MCF-7 cells were infected with a GFP-LC3-expressing adenovirus. MCF-7 cell proliferation was inhibited and autophagy was activated in the same dose-dependent manner. Bortezomib induced a dose-dependent increase in LC3-II. However, when MCF-7 cells were co-treated with bortezomib and 3-MA, 3-MA blocked the increase in LC3-II protein expression and led to a significant inhibition of cell proliferation. Inhibition of the proteasome may induce autophagy in human breast cancer MCF-7 cells and 3-MA could inhibit autophagy induced by the proteasome inhibitor. A combination of 3-MA and bortezomib increases cell death. These findings indicate that suppression of the two intracellular degradation systems may shed new light on breast cancer control.
\end{abstract}

Correspondence to: Dr Shengrong Sun, Department of Breast and Thyroid Surgery, Renmin Hospital of Wuhan University, Wuhan, Hubei 430060, P.R. China

E-mail: sun137@sina.com

*Contributed equally

Key words: proteasome inhibitors, autophagy, cell death

\section{Introduction}

Protein degradation is an essential cellular function that, when dysregulated or impaired, may lead to a wide variety of disease states, such as cancer. The ubiquitin-proteasome system (UPS) and the autophagy-lysosome are the two major intracellular protein degradation systems $(1,2)$. The UPS serves as the primary route of degradation for thousands of short-lived proteins (3). UPS-mediated catabolism is essential to maintain amino acid pools in acute starvation and contributes significantly to the degradation of defective proteins (4-6). Autophagy is primarily responsible for degrading long-lived proteins and maintaining amino acid pools in the setting of chronic starvation. Autophagy also plays a housekeeping role in removing misfolded or aggregated proteins, clearing damaged organelles and eliminating intracellular pathogens. Thus, autophagy is generally thought of as a survival mechanism (7).

Inhibition of proteasome activity has become a new chemotherapy strategy and a number of chemicals and natural compounds have proven to be effective at inducing tumor cell death by inhibiting proteasome activity $(8,9)$. Currently, one proteasome inhibitor, bortezomib (Velcade ${ }^{\circledR}$ ), has been approved for treating relapsed multiple myeloma. Autophagy induction is greatly altered in cancer tissue. The tumor microenvironment is commonly poor in nutrients and oxygen, favoring autophagy induction (10). However, human cancer frequently displays inactivating mutations in pro-autophagy genes and the activation of anti-autophagy genes $(10,11)$. These changes contribute differentially to the autophagic capacity in tumors, depicting the duality of autophagy in cancer.

In the present study, we investigated whether the proteasome inhibitor bortezomib induces autophagy in human MCF-7 breast cancer cells. We showed that treatment of MCF-7 cells with bortezomib induced autophagy. Furthermore, inhibition of autophagy by the autophagy inhibitor 3-MA enhanced inhibition of cell proliferation by bortezomib alone. These findings indicated the benefits of suppressing the two cellular degradation systems as a novel tumor-specific therapeutic strategy in breast cancer.

\section{Materials and methods}

Reagents. DMEM was from Thermol Scientific (Waltham, MA, USA), fetal bovine serum (FBS) was from Atlantic Biologicals (Lawrenceville, GA, USA), bortezomib was 
from LC Laboratories (Woburn, MA, USA), 3-MA was from Sigma-Aldrich (St. Louis, MO, USA) and the cell proliferation reagent WST-1 was from Roche (Mannheim, Germany). All other chemicals were from Sigma-Aldrich or Invitrogen (Carlsbad, CA, USA).

The following antibodies were used. Horseradish peroxidase-conjugated goat anti-mouse/rabbit secondary antibodies were from Santa Cruz Biotechnology (Santa Cruz, CA, USA), anti- $\beta$-catenin was from BD Transduction Laboratories (San Diego, CA, USA), anti-LC3 was from MBL Co. (Woburn, MA, USA) and anti-GAPDH was from Sigma.

Cell line and culture. The human breast cancer cell line, MCF-7, was obtained from the American Type Culture Collection (ATCC) (Manassas, VA, USA). MCF-7 cells were maintained in DMEM supplemented with $10 \%$ of FBS, 4 mM L-glutamine and $1 \mathrm{X}$ penicillin/streptomycin under standard conditions of temperature $\left(37^{\circ} \mathrm{C}\right)$ and carbon dioxide (5\%). Cells in the mid-log phase were used in the experiments.

Cell proliferation assay. According to the procedure described in the data sheet of WST-1, MCF-7 cells were plated at a density of 10,000 cells per $100 \mu \mathrm{l}$ per well in 96 -well plates. Following treatment, the absorbance of the samples was measured at $450 \mathrm{~nm}$ with a Vmax kinetic microplate reader (Molecular Devices, Sunnyvale, CA, USA), using a background control as a blank. The reference wavelength was $650 \mathrm{~nm}$.

Fluorescence microscopy for GFP-LC3 puncta. For GFP-LC3 redistribution, MCF-7 cells were infected with a GFP-LC3-expressing adenovirus. At $24 \mathrm{~h}$ post-infection, cells were treated with the drugs. GFP-LC3 was detected using a fluorescence microscope (Axiovert 200M, Carl Zeiss Light Microscopy, Göttingen, Germany). The percentage of GFP-LC3-positive cells with punctate staining was determined in three independent experiments. To quantify the number of GFP-LC3-positive autophagosomes per transfected cell, six random fields representing 200-300 cells were counted.

Western blotting. MCF-7 cells were washed twice with cold phosphate-buffered saline (PBS) and lysed with 1X loading buffer and a proteasome inhibitor cocktail. The total proteins were extracted for Western blotting. The concentration of proteins for each sample was determined using the bicinchoninic acid protein assay kit (Pierce Biotechnology, Rockford, IL, USA). Equal amounts of protein (30 $\mu \mathrm{g} /$ lane) were resolved by $14 \%$ SDS-PAGE and electrically transferred onto polyvinylidene difluoride (PVDF) membranes (Bio-Rad, Hercules, CA, USA). The membranes were blocked with $5 \%$ milk in PBS-T for $1 \mathrm{~h}$ at room temperature and then incubated overnight at $4^{\circ} \mathrm{C}$ with the following primary antibodies: anti- $\beta$-catenin (1:2000) and anti-LC3 (1:1000). The membranes were then incubated with horseradish peroxidase-conjugated goat anti-mouse/ rabbit secondary antibodies for $60 \mathrm{~min}$ at room temperature. ECL-Plus detection reagents (GE Healthcare, Piscataway, NJ, USA) and a VersaDoc imaging system (model 3000, Bio-Rad) were used to visualize and digitalize the Western blotting images. The densitometry of the Western blotting was determined with Quantity One software (Bio-Rad). The same-lane GAPDH level was employed as a protein loading control.
A

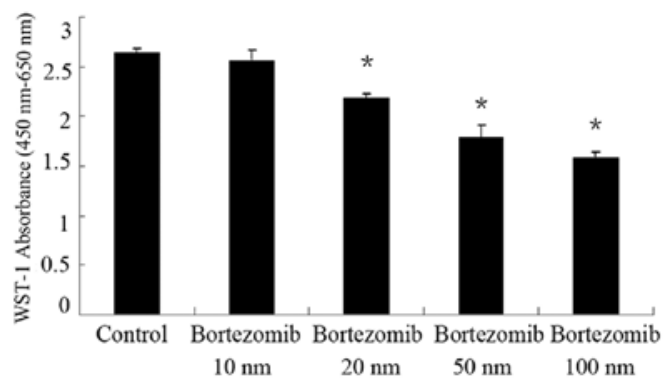

B

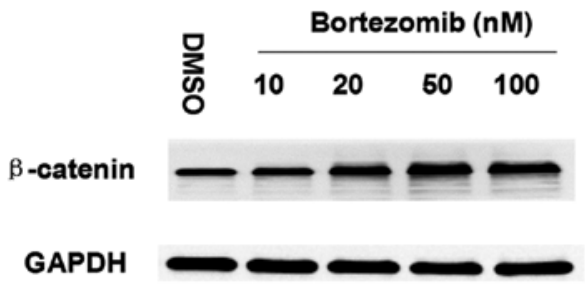

C

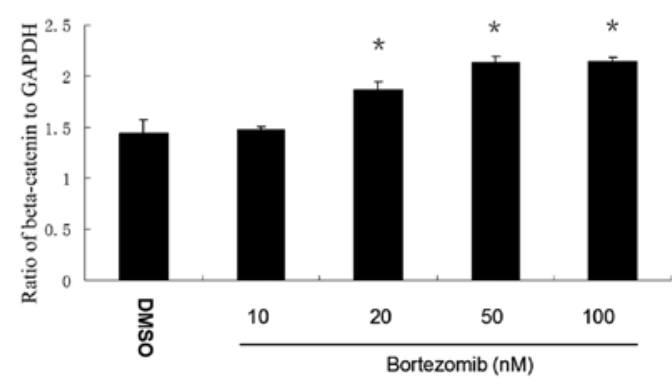

Figure 1. Inhibition of MCF-7 cell proliferation and proteasome activity by the proteasome inhibitor bortezomib. (A) Treatment with bortezomib for 48 $\mathrm{h}$ dose-dependently lowered cell proliferation as determined by MTT assay. (B) Bortezomib blocked the degradation of the endogenous proteasome substrate $\beta$-catenin in MCF-7 cells. (C) Relative amounts of $\beta$-catenin were described as the ratio of $\beta$-catenin to GAPDH. ${ }^{*} \mathrm{P}<0.01$ compared with the control group.

Statistical analysis. Experimental data are shown as the mean \pm SD. Statistical comparisons were carried out with the Student's t-test using SPSS 11.0 software. $\mathrm{P}<0.05$ was considered to denote statistical significance.

\section{Results}

Bortezomib inhibited cell proliferation and proteasome activity. After MCF-7 cells were treated with bortezomib at the indicated concentrations for $48 \mathrm{~h}$, cell viability was assessed by MTT assay. MCF-7 cell proliferation was inhibited by bortezomib in a dose-dependent manner (Fig. 1A). Compared with the control, 48-h treatment of bortezomib at a dose of $50 \mathrm{nM}$ inhibited MCF-7 cell proliferation by approximately $32 \%$.

To study the effect of blockade of UPS on MCF-7 cells by bortezomib, Western blot analysis was performed. $\beta$-catenin is an endogenous proteasome substrate (12). Inhibition of proteasome activity by bortezomib was confirmed by accumulation of this known proteasome substrate. After the MCF-7 cells were treated with bortezomib at the indicated concentrations for $48 \mathrm{~h}, \beta$-catenin dramatically increased (Fig. 1B and C).

Bortezomib induced autophagy. As the amount of LC3 protein, particularly LC3-II, correlates with the extent of 
$\mathbf{A}$

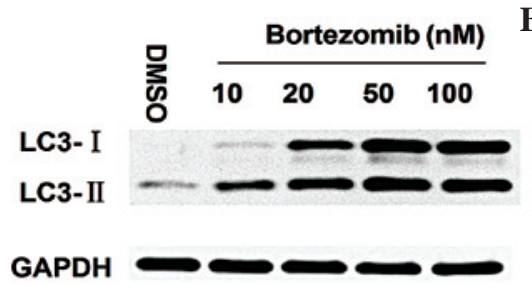

B

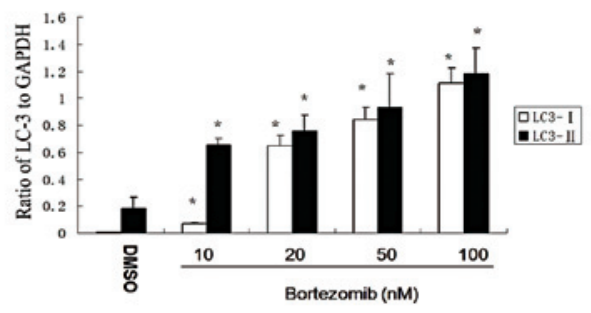

C

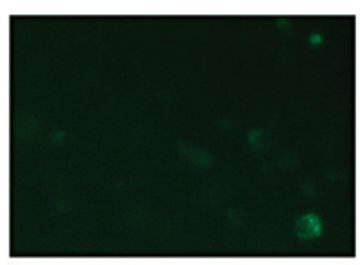

Vehicle control (DMSO)

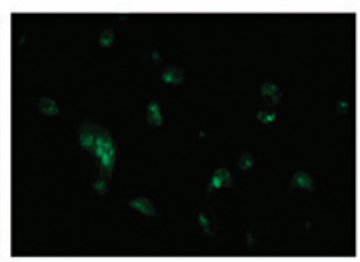

24h Bortezomib (50 $\mathrm{nM})$

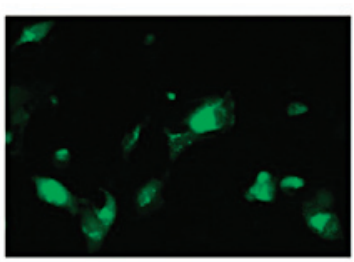

48h Bortezomib (50 $\mathrm{nM})$

D

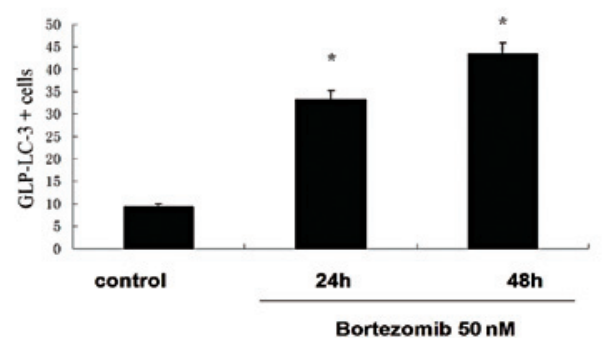

Figure 2. Induction of autophagy by bortezomib. (A) Bortezomib dose-dependently increased the protein expression of LC3-I and -II. (B) Relative amounts of LC3-I and LC3-II described as ratios of LC3-I and LC3-II to GAPDH. (C) MCF-7 cells were first infected with Ad-GFP-LC3 for $24 \mathrm{~h}$ and then treated with vehicle control, bortezomib $(50 \mathrm{nM})$ for $24 \mathrm{~h}$, or bortezomib $(50 \mathrm{nM})$ for $48 \mathrm{~h}$. Cells were then analyzed by fluorescence microscopy for LC3 translocation The percentages of GFP-LC3-positive cells showing puncta were determined (columns, mean; bars, SD). (D) The percentage of GFP-LC3-positive cells in each group. " $\mathrm{P}<0.01$ compared with the control group.

A

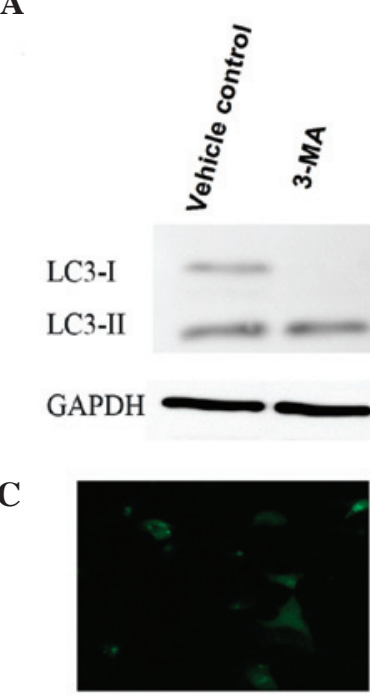

Vehicle control

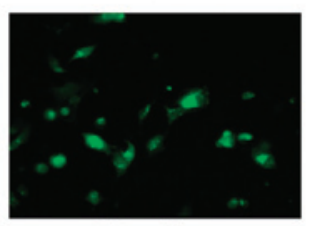

Bortezomib
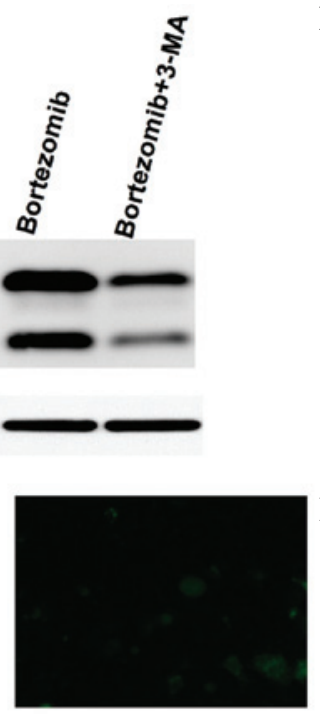

3-MA

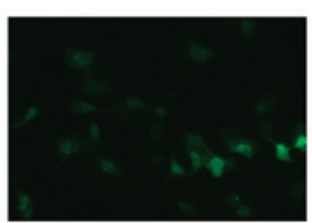

Bortezomib+3-MA
B

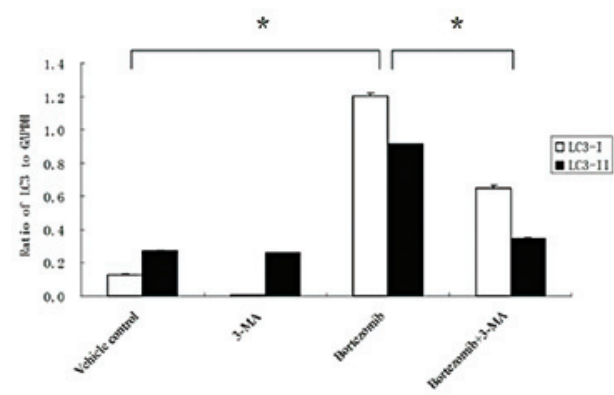

D

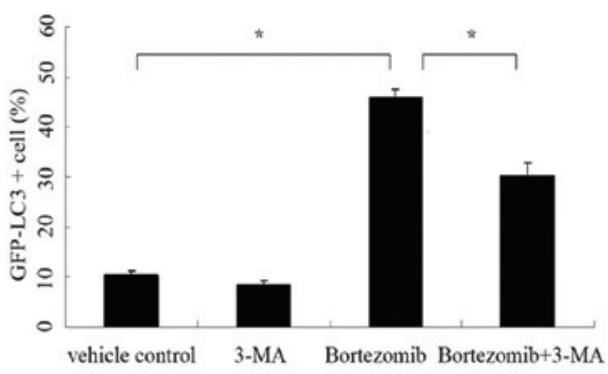

Figure 3. Blockade of bortezomib-induced autophagy by 3-MA. (A) 3-MA blocked the increase in LC3-II protein expression induced by bortezomib. (B) Relative amounts of LC3-I and LC3-II, described as ratios of LC3-I and LC3-II to GAPDH. (C) MCF-7 cells were first infected with Ad-GFP-LC3 for $24 \mathrm{~h}$ and then treated with vehicle control, bortezomib $(50 \mathrm{nM}), 3-\mathrm{MA}(10 \mathrm{mM})$,or a combination of bortezomib $(50 \mathrm{nM})$ and $3-\mathrm{MA}(10 \mathrm{mM})$ for $48 \mathrm{~h}$. Cells were then analyzed by fluorescence microscopy for LC3 translocation. The percentages of GFP-LC3-positive cells showing puncta were determined (columns, mean; bars, SD). (D) The percentage of GFP-LC3-positive cells in each group. " $\mathrm{P}<0.01$. 
*

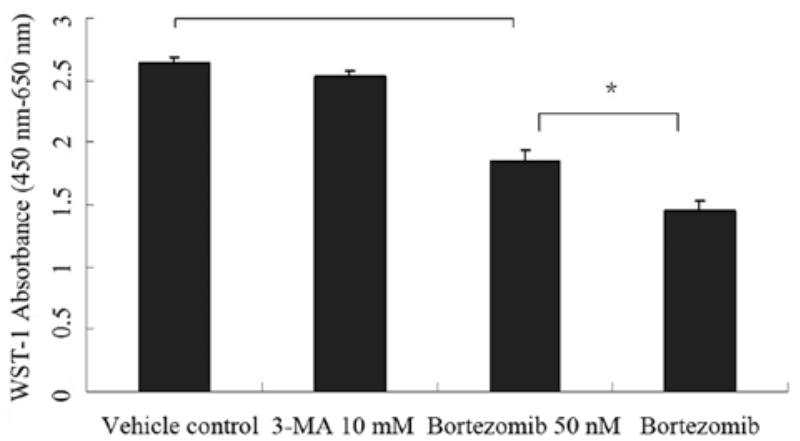

$50 \mathrm{nM}+3-\mathrm{MA} 10 \mathrm{nM}$

Figure 4. Enhancement of the effects of bortezomib on cell proliferation by 3-MA. Result of MTT assays. Compared with the bortezomib group, cell viability decreased significantly at $48 \mathrm{~h}$ after co-treatment with 3 -MA. ${ }^{*} \mathrm{P}<0.01$.

autophagy, the effect of bortezomib on LC3 protein expression and the alteration of GFP-LC3 punctation in MCF-7 cells were studied. Treatment of MCF-7 cells with bortezomib induced a dose-dependent increase in LC3-II (Fig. 2A and B) and a time-dependent accumulation of GFP-LC3 puncta (Fig. 2C and D).

3-MA blocked autophagy induced by bortezomib. To determine whether the autophagy induced by bortezomib is blocked by 3-MA, we co-treated the MCF-7 cells with bortezomib and 3-MA. We showed that 3-MA blocked the increase in LC3-II protein expression (Fig. 3A and B) and the accumulation of GFP-LC3 puncta induced by bortezomib (Fig. 3C and D), indicating that 3-MA blocked the autophagy induced by bortezomib.

To determine whether the antitumor effects of bortezomib are enhanced by the modulation of autophagy, MCF-7 cells were treated with the combination of bortezomib and 3-MA at the indicated concentrations for $48 \mathrm{~h}$, and cell viability was assessed by MTT assay.

Bortezomib alone retarded cell proliferation compared with the control group (Fig. 4). However, the combination of bortezomib and 3-MA led to a significant inhibition of MCF-7 cell proliferation, indicating that suppression of the proteasome together with the compensatory autophagy caused maximal stress and demise to cancer cells.

\section{Discussion}

Recent studies have shown that proteasome inhibitors serve as a new and promising class of anticancer agents (13-17). In this study we showed that MCF-7 cell proliferation was inhibited by bortezomib in a dose-dependent manner. Furthermore, we found that autophagy was activated after MCF-7 cells were incubated with bortezomib as evidenced by the increased percentages of GFP-LC3-positive cells showing puncta, and up-regulation of LC3-II protein.

Recent studies have reported that impairment of the UPS leads to increased autophagic function (18-20). They revealed interaction between the UPS and autophagy $(18,21)$. Autophagy is a self-degradative process that is crucial for balancing sources of energy at critical times in development and in response to nutrient stress. It is also an evolutionarily conserved self-digestion process responsible for the turnover of whole organelles and macromolecules $(22,23)$. It is possible that autophagy induced by the inhibition of proteasome function may serve as a compensatory mechanism in response to protein accumulation to alleviate the toxicity of proteasome inhibitors $(20,24)$, suggesting a coordinated and complementary relationship between these degradation systems.

To determine whether the autophagy induced by bortezomib is blocked by the autophagy inhibitor 3-MA, we co-treated the MCF-7 cells with bortezomib and 3-MA. We showed that 3-MA blocked the autophagy induced by bortezomib. Moreover, to determine whether the antitumor effects of bortezomib are enhanced by the modulation of autophagy, MCF-7 cells were treated with the combination of bortezomib and 3-MA, and cell viability was assessed by MTT assay. Notably, 3-MA alone also had an inhibitory effect; however, the combination of bortezomib and 3-MA led to a significant inhibition of MCF-7 cell proliferation, indicating that suppression of the proteasome together with the compensatory autophagy caused maximal stress and demise to cancer cells.

Single use of proteasome inhibitors may not be effective for resistant myeloma and for solid tumors (25-27). A number of regimes have been examined that suppress autophagy to enhance cancer cell death (28-30). It appears that simultaneous inhibition of autophagy and the proteasome could offer a unique advantage based on this study.

It has to be pointed out that suppressing autophagy is not a generic strategy to enhance therapeutic efficacy for all types of cancer. In certain cases, cytotoxic effects, rather than cytoprotective effects, were shown to be caused by autophagy (10).

In conclusion, we provide evidence that the proteasome inhibitor bortezomib produces in vitro growth inhibition in human MCF-7 cells, and inhibition of the proteasome activates autophagy. Moreover, we demonstrated that 3-MA blocked the autophagy induced by bortezomib and the combined use of bortezomib and 3-MA enhanced cell death.

Whether these findings can be translated into chemoprophylactic and therapeutic options for the treatment of breast cancers, however, awaits further clinical investigation.

\section{References}

1. Goldberg AL: Protein degradation and protection against misfolded or damaged proteins. Nature 426: 895-899, 2003.

2. Rubinsztein DC: The roles of intracellular protein-degradation pathways in neurodegeneration. Nature 443: 780-786, 2006.

3. Ciechanover A, Orian A and Schwartz AL: Ubiquitin-mediated proteolysis: biological regulation via destruction. Bioessays 22: 442-451, 2000

4. Wheatley DN and Inglis MS: An intracellular perfusion system linking pools and protein synthesis. J Theor Biol 83: 437-445, 1980.

5. Vabulas RM and Hartl FU: Protein synthesis upon acute nutrient restriction relies on proteasome function. Science 310: 1960-1963, 2005.

6. Ciechanover A and Brundin P: The ubiquitin proteasome system in neurodegenerative diseases: sometimes the chicken, sometimes the egg. Neuron 40: 427-446, 2003.

7. Pan T, Kondo S, Zhu W, Xie W, Jankovic J and Le W: Neuroprotection of rapamycin in lactacystin-induced neurodegeneration via autophagy enhancement. Neurobiol Dis 32: 16-25, 2008. 
8. Momose I, Lijima M, Kawada M and Ikeda D: A new proteasome inhibitor, TP-110, induces apoptosis in human prostate cancer PC-3 cells. Biosci Biotechnol Biochem 71: 1036-1043, 2007.

9. Yang H, Chen D, Cui QC, Yuan X and Dou QP: Celastrol, a triterpene extracted from the Chinese, 'Thunder of God Vine', is a potent proteasome inhibitor and suppresses human prostate cancer growth in nude mice. Cancer Res 66: 4758-4765, 2006.

10. Mathew R, Karantza-Wadsworth V and White E: Role of autophagy in cancer. Nat Rev Cancer 7: 961-967, 2007.

11. Gozuacik D and Kimchi A: Autophagy as a cell death and tumor suppressor mechanism. Oncogene 23: 2891-2906, 2004.

12. Liu J, Zheng H, Tang M, Ryu YC and Wang X: A therapeutic dose of doxorubicin activates ubiquitin-proteasome system-mediated proteolysis by acting on both the ubiquitination apparatus and proteasome. Am J Physiol Heart Circ Physiol 295: H2541-H2550, 2008.

13. Richardson PG, Mitsiades C, Hideshima T and Anderson KC: Bortezomib: proteasome inhibition as an effective anticancer therapy. Annu Rev Med 57: 33-47, 2006.

14. Cusack JC: Rationale for the treatment of solid tumors with the proteasome inhibitor bortezomib. Cancer Treat Rev 29 (Suppl 1): 21-31, 2003.

15. Orlowski RZ and Dees EC: The role of the ubiquitination-proteasome pathway in breast cancer: applying drugs that affect the ubiquitin-proteasome pathway to the therapy of breast cancer. Breast Cancer Res 5: 1-7, 2003.

16. Mitsiades N, Mitsiades CS, Richardson PG, et al: The proteasome inhibitor PS-341 potentiates sensitivity of multiple myeloma cells to conventional chemotherapeutic agents: therapeutic applications. Blood 101: 2377-2380, 2003.

17. Small GW, Shi YY, Edmund NA, Somasundaram S, Moore DT and Orlowski RZ: Evidence that mitogen-activated protein kinase phosphatase-1 induction by proteasome inhibitors plays an antiapoptotic role. Mol Pharmacol 66: 1478-1490, 2004

18. Pandey UB, Nie Z, Batlevi Y, et al: HDAC6 rescues neurodegeneration and provides an essential link between autophagy and the UPS. Nature 447: 859-863, 2007.
19. Ding WX, Ni HM, Gao W, et al: Linking of autophagy to ubiquitin-proteasome system is important for the regulation of endoplasmic reticulum stress and cell viability. Am J Pathol 171: 513-524, 2007.

20. Iwata A, Riley BE, Johnston JA and Kopito RR: HDAC6 and microtubules are required for autophagic degradation of aggregated huntingtin. J Biol Chem 280: 40282-40292, 2005

21. Kirkin V, Lamark T, Sou YS, et al: A role for NBR1 in autophagosomal degradation of ubiquitinated substrates. Mol Cell 33: 505-516, 2009.

22. Mizushima N, Levine B, Cuervo AM and Klionsky DJ: Autophagy fights disease through cellular self-digestion. Nature 451: 1069-1075, 2008

23. Levine B and Kroemer G: Autophagy in the pathogenesis of disease. Cell 132: 27-42, 2008.

24. Korolchuk VI, Menzies FM and Rubinsztein DC: Mechanisms of cross-talk between the ubiquitin-proteasome and autophagy-lysosome systems. FEBS Lett 584: 1393-1398, 2010.

25. Chauhan D, Hideshima T and Anderson KC: Proteasome inhibition in multiple myeloma: therapeutic implication. Annu Rev Pharmacol Toxicol 45: 465-476, 2005.

26. Aghajanian C, Soignet S, Dizon DS, et al: A phase I trial of the novel proteasome inhibitor PS341 in advanced solid tumor malignancies. Clin Cancer Res 8: 2505-2511, 2002.

27. Papandreou CN and Logothetis CJ: Bortezomib as a potential treatment for prostate cancer. Cancer Res 64: 5036-5043, 2004.

28. Degenhardt K, Mathew R, Beaudoin B, et al: Autophagy promotes tumor cell survival and restricts necrosis, inflammation, and tumorigenesis. Cancer Cell 10: 51-64, 2006.

29. Amaravadi RK, Yu D, Lum JJ, et al: Autophagy inhibition enhances therapy-induced apoptosis in a Myc-induced model of lymphoma. J Clin Invest 117: 326-336, 2007.

30. Carew JS, Nawrocki ST, Kahue CN, et al: Targeting autophagy augments the anticancer activity of the histone deacetylase inhibitor SAHA to overcome Bcr-Abl-mediated drug resistance. Blood 110: 313-322, 2007. 